\title{
A Reptation Model for Magnetic Materials
}

\author{
Thomas R. Braun*, Ralph C. Smith ${ }^{\dagger}$ and Marcelo J. Dapino ${ }^{\ddagger}$ \\ * Center for Research in Scientific Computation, North Carolina State Univ., Raleigh, NC 27695 \\ ${ }^{\dagger}$ Department of Mechanical Engineering, The Ohio State University, Columbus, OH 43210
}

\begin{abstract}
Reptation, or accommodation, is manifested in ferromagnetic materials in a variety of operating regimes and hence must be incorporated in models used for comprehensive material characterization or model-based control design. Because the microscopic mechanisms which cause reptation are complex, we characterize the effect in a phenomenological macroscopic manner within the context of a homogenized energy framework for ferromagnetic hysteresis. Attributes of the model are illustrated through comparison with experimental data.
\end{abstract}

Keywords: Reptation, hysteresis, homogenized energy framework, ferromagnetic materials

\section{Introduction}

Reptation in ferromagnetic materials is the phenomenon in which repeated biased minor hysteresis loops with identical input fields yield output inductions/magnetizations that shift with each loop. This is also referred to as accommodation and is shown for a nickel rod in Figure 1. Each shift is less than the previous one so that the material approaches a steady-state condition if enough loops are performed. However, this steadystate is temporary since upon traversing a major loop and returning to the previous input level, reptation is again observed. For this reason, reptation cannot be "trained" out of a material, and must be included in any mathematical model when high precision is needed.

The physical causes of reptation are not well understood. Although the behavior bears a resemblance to thermally-induced creep or after-effects $[2,3,4]$, it cannot be fully explained by thermal effects alone. It is believed to be related to moment interaction fields, but these themselves are typically only described in a phenomenological manner. Hence reptation has been characterized in a phenomenological manner as a combination of historydependent and history-independent Preisach models [8]. Here we take an alternative approach in which we relate reptation to the underlying energy of the material. This is done within the context of the homogenized energy model, where energy principles on a lattice level and statistical distributions are combined to provide a macroscopic model.

The homogenized energy framework for ferromagnetic hysteresis is summarized in Section 2 and extended in Section 3 to incorporate reptation. Attributes of the extended model are illustrated in Section 4 where the model response is compared with experimental data from a nickel rod.

\section{Homogenized Energy Framework}

As detailed in $[2,5,6,7]$, the homogenized energy model for ferromagnetic materials is

$$
[M(H)](t)=\int_{0}^{\infty} \int_{-\infty}^{\infty}\left[\bar{M}\left(H+H_{I} ; H_{c}\right)\right](t) \nu_{c}\left(H_{c}\right) \nu_{I}\left(H_{I}\right) d H_{I} d H_{c}
$$

\footnotetext{
*Email: trbraun@ncsu.edu; Telephone: 919-854-2746

${ }^{\dagger}$ Email: rsmith@eos.ncsu.edu; Telephone: 919-515-7552

‡Email: dapino.1@osu.edu; Telephone: 614-688-3689
} 

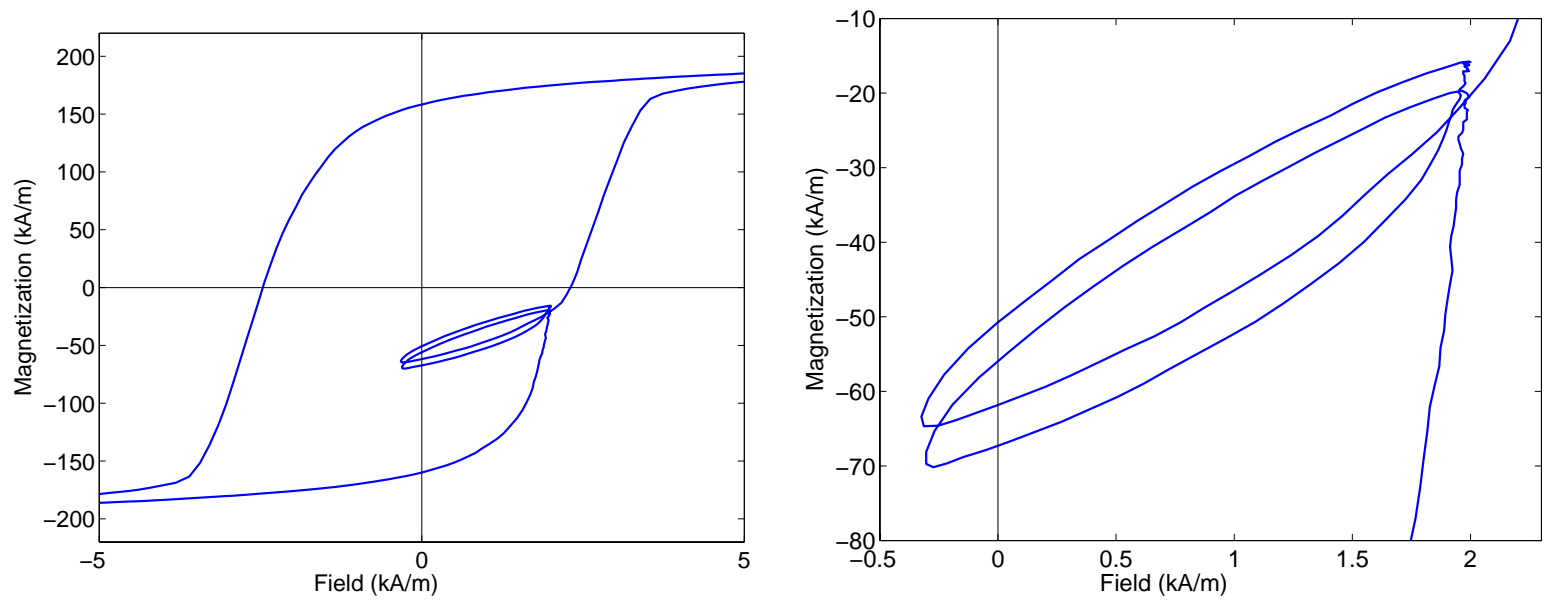

Figure 1: Magnetic field versus magnetization for a steel rod. Both minor loops were produced with identical input field levels and the shift in the resulting magnetization is due to reptation effects.

where $H$ is the input magnetic field, $M$ is the macroscopic magnetization, $H_{c}$ and $H_{I}$ are local coercive and interaction fields, and $\nu_{c}, \nu_{I}$ are associated densities which incorporate the effects of material nonhomogeneities and variable effective fields $\left(H_{e}=H+H_{I}\right)$. The integrals are typically approximated using Gaussian or NewtonCotes quadrature techniques. To illustrate, let $H_{c}[i], i=1, \ldots, N_{c}$ and $H_{I}[j], j=1, \ldots, N_{I}$ denote the abscissas (quadrature points) and $\widehat{w}_{c}[i], \widehat{w}_{I}[j]$ denote the respective quadrature weights. Approximation of the integrals in (1) then yields the discretized model

$$
\begin{aligned}
{[M(H)](t) } & =\sum_{i=1}^{N_{c}} \sum_{j=1}^{N_{I}}\left[\bar{M}\left(H+H_{I}[j] ; H_{c}[i]\right)\right](t) \nu_{c}\left(H_{c}[i]\right) \nu_{I}\left(H_{I}[j]\right) \widehat{w}_{c}[i] \widehat{w}_{I}[j] \\
& =\sum_{i=1}^{N_{c}} \sum_{j=1}^{N_{I}}\left[\bar{M}\left(H+H_{I}[j] ; H_{c}[i]\right)\right](t) w_{c}[i] w_{I}[j],
\end{aligned}
$$

where $w_{c}[i]=\widehat{w}_{c}[i] \nu_{c}\left(H_{c}[i]\right)$ and $w_{I}[j]=\widehat{w}_{I}[j] \nu_{I}\left(H_{I}[j]\right)$.

The kernel $\bar{M}$ is formulated by balancing the Gibbs and relative thermal energies. For simplicity, we assume that the operating temperature of the device is approximately constant. For the one-dimensional model, the Gibbs energy is then given by

$$
G=\psi(M)-\mu_{0} H M
$$

where $\mu_{0}$ is the permeability of free space, and the Helmholtz energy is

$$
\psi(M)= \begin{cases}\frac{1}{2} \eta\left(M+M_{R}\right)^{2}, & M \leq-M_{I} \\ \frac{1}{2} \eta\left(M-M_{R}\right)^{2}, & M \geq M_{I} \\ \frac{1}{2} \eta\left(M_{I}-M_{R}\right)\left(\frac{M^{2}}{M_{I}-M_{R}}\right), & |M|<M_{I} .\end{cases}
$$

Here $M_{R}$ denotes the remanence magnetization, $M_{I}$ is the inflection point and $\eta$ is the approximate slope $\frac{d M}{d H}$ after switching occurs. The Gibbs energy is balanced with the thermal energy via the Boltzmann relation

$$
\mu(G)=C e^{-G(H, M) V / k T}
$$

where $V$ is a reference volume, $T$ is the temperature in degrees Kelvin, $k$ is Boltzmann's constant, and $C$ is 
chosen to ensure integration to unity. As detailed in [5], this yields the average magnetization relations

$$
\left\langle M_{+}\right\rangle=\frac{\int_{M_{0}}^{\infty} M e^{-G(H, M) V / k T} d M}{\int_{M_{0}}^{\infty} e^{-G(H, M) V / k T} d M}, \quad\left\langle M_{-}\right\rangle=\frac{\int_{-\infty}^{M_{0}} M e^{-G(H, M) V / k T} d M}{\int_{-\infty}^{M_{0}} e^{-G(H, M) V / k T} d M}
$$

for positively and negatively oriented moments, respectively. In practice, the positive or negative inflection point $\left( \pm M_{I}\right)$ is substituted for $M_{0}$ for computational efficiency.

Let $x_{+}$denote the fraction of magnetic moments oriented in the positive direction. Then $x_{-}=1-x_{+}$ moments are oriented negatively, and the kernel or hysteron is given by

$$
\bar{M}=x_{+}\left\langle M_{+}\right\rangle+\left(1-x_{+}\right)\left\langle M_{-}\right\rangle .
$$

The moment fraction $x_{+}$evolves according to the differential equation

$$
\dot{x}_{+}=-p_{+-} x_{+}+p_{-+}\left(1-x_{+}\right)
$$

where $p_{+-}$denotes the likelihood of a moment switching from positive to negative, and $p_{-+}$is the likelihood of switching from positive to negative. These likelihoods are quantified by the relations

$$
p_{+-}=\frac{1}{\mathcal{T}} \frac{\int_{M_{I}}^{M_{I}+\epsilon} e^{-G(H, M) V / k T} d M}{\int_{M_{I}}^{\infty} e^{-G(H, M) V / k T} d M}, \quad p_{-+}=\frac{1}{\mathcal{T}} \frac{\int_{-M_{I}-\epsilon}^{M_{I}} e^{-G(H, M) V / k T} d M}{\int_{-\infty}^{-M_{I}} e^{-G(H, M) V / k T} d M},
$$

where $\mathcal{T}$ denotes the material-dependent relaxation time (i.e., $\omega=\frac{1}{\mathcal{T}}$ quantifies the frequency at which moments attempt to switch) and $\epsilon$ is a small positive constant. The value of $\epsilon$ is arbitrary so long as it is significantly smaller than $P_{I}$. However, the values of $\mathcal{T}$ and $V$ implicitly depend on $\epsilon$. Thus, the integral does not limit to 0 as $\epsilon$ decreases. As detailed in [5], point evaluation $\left(e^{-G\left(H, M_{I}\right) V / k T}\right)$ in the numerator is sometimes employed rather than the integrals containing $\epsilon$, especially in higher dimensions. This yields nearly identical model predictions but must be interpreted as approximate from a statistical sense since the likelihood of any point occurring within a continuum is 0 .

\subsection{Theoretical Model}

\section{Reptation Model}

The physical causes of reptation are not well understood but are known to be dependent on the electron spins of the magnetic moments. Repeated minor loops over the same input field lessen the spin, which in turn changes the level of interaction between a given moment and its neighbors. Thus, reptation is tied to the behavior of the interaction density $\nu_{I}$. However, this does not in itself give sufficient detail to implement a model. As such, we impose the empirical constraints:

- reptation behavior approaches a steady-state response,

- reptation behavior resets so that after a major loop, any previous training is lost, and

- reptation behavior is dependent on input field levels; training at one input level has no effect on reptation amounts at other input levels.

To this end, reptation is considered by changing the density $\nu_{I}$ in (1) and (2). Let $\nu_{n o m}$ represent the nominal or steady-state interactive field density. This is identified as a model parameter in the same manner $\nu_{I}$ was considered previously. The field-dependent density is then defined as

$$
\nu_{I}(H)=\frac{\nu_{\text {nom }}\left(\frac{H}{(1+\alpha)^{\lambda^{k}}}\right)}{(1+\alpha)^{\lambda^{k}}}
$$


where $\alpha \geq 0$ and $\lambda \in[0,1)$ are model parameters and $k \in \mathbb{N}$ is an internal counter. That is, we define the interaction field density as a scaled multiple of the nominal interaction field density. This scaled version is depicted for various $k$ values in Figure 2 and in appearance is similar to stretching the density. The denominator in (10) assures that the area under the density remains constant as $k$ changes - the overall amount of influence exerted by a moment on others is constant but this influence becomes more localized as reptation increases. The additional two parameters $\alpha$ and $\lambda$ quantify the difference between the nominal and beginning $(k=1)$ densities and the rate at which $\nu_{I}$ approaches $\nu_{n o m}$. Note that other methods of convergence to a nominal density are possible - e.g., a normal density whose variance decreases to some constant value — but (10) is chosen here for its application to general densities and its computational simplicity.

The requirement that reptation be related to moment interactions and that behavior approach a steady state are satisfied in (10). The remaining two requirements effect the choice of $k$. Reptation behavior is dependent on input field levels - the behavior at one field level is independent of that given at another. Thus, $k$ must be a function of the input field $H$. We let $k$ be a counter over $H$ :

$$
k(H)=\text { number of times input level has passed through a value of } H \text {. }
$$

Note that $k(H) \geq 1$ whenever it is employed. The evolution of (11) is depicted for an example input in Figure 3 .

It is apparent that $k(H)$ is discontinuous which introduces a discontinuity in the density (10). However, it important to note that this discretization does not introduce discontinuities in the magnetization. Consider for example the increasing $H$ portion of a major loop together with several minor loops. As $\nu_{I}$ approaches $\nu_{n o m}$ the effective field approaches the applied field. A negatively oriented moment may in the process cross its coercive point and switch to positive. However, as long as the applied field is greater than the negative coercive point, no positively oriented moment will switch to negative. Thus, the magnetization shifts upward as $k$ increases, i.e., as minor loops are traversed. The only change to the magnetization results from some moments switching earlier than they would have otherwise. A more interesting case occurs when leaving minor loops. Here $k$ drops to a lower value (presumably 1). The interaction field increases when this occurs. No moments will switch from negative to positive at this point — any whose effective field is moved above the coercive point in this process would have already switched when entering the first minor loop — but a few moments that switched positive may have an effective field that goes sufficiently negative to cross the negative coercive point and switch back to negative orientation. This would result in a magnetization that exits the minor loops at a value below what it obtained in the minor loops. This prediction is observed in data as can be seen in Figure 1.

Behavior may be reset by setting $k(H)=0$ for all $H$ before the increment is performed. The empirical constraints do not specify when such a reset occurs, only that it must occur at least once per major loop. However, the reset is by nature an irreversible behavior. The homogenized energy framework includes one other

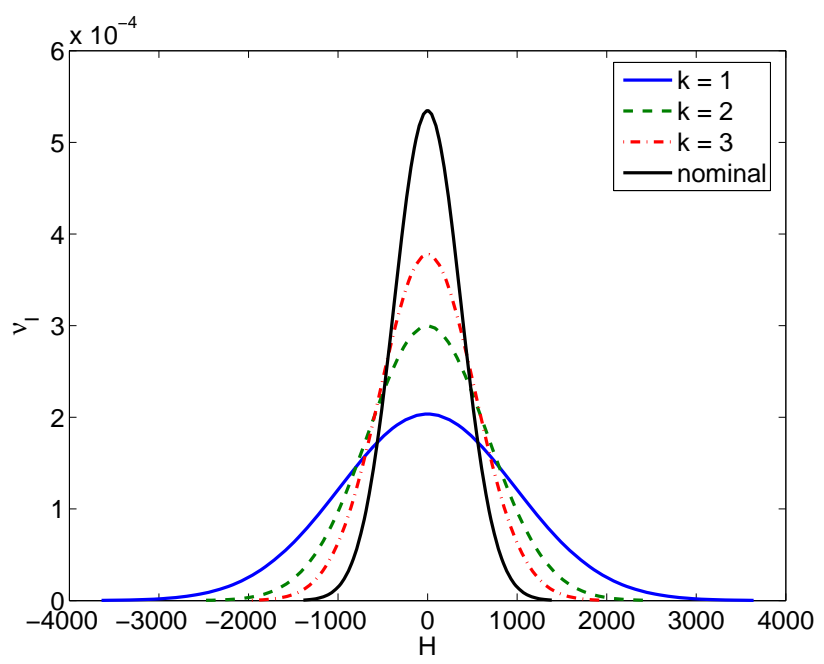

Figure 2: Distribution $\nu_{I}$ given in (10) as a function of $k$. 


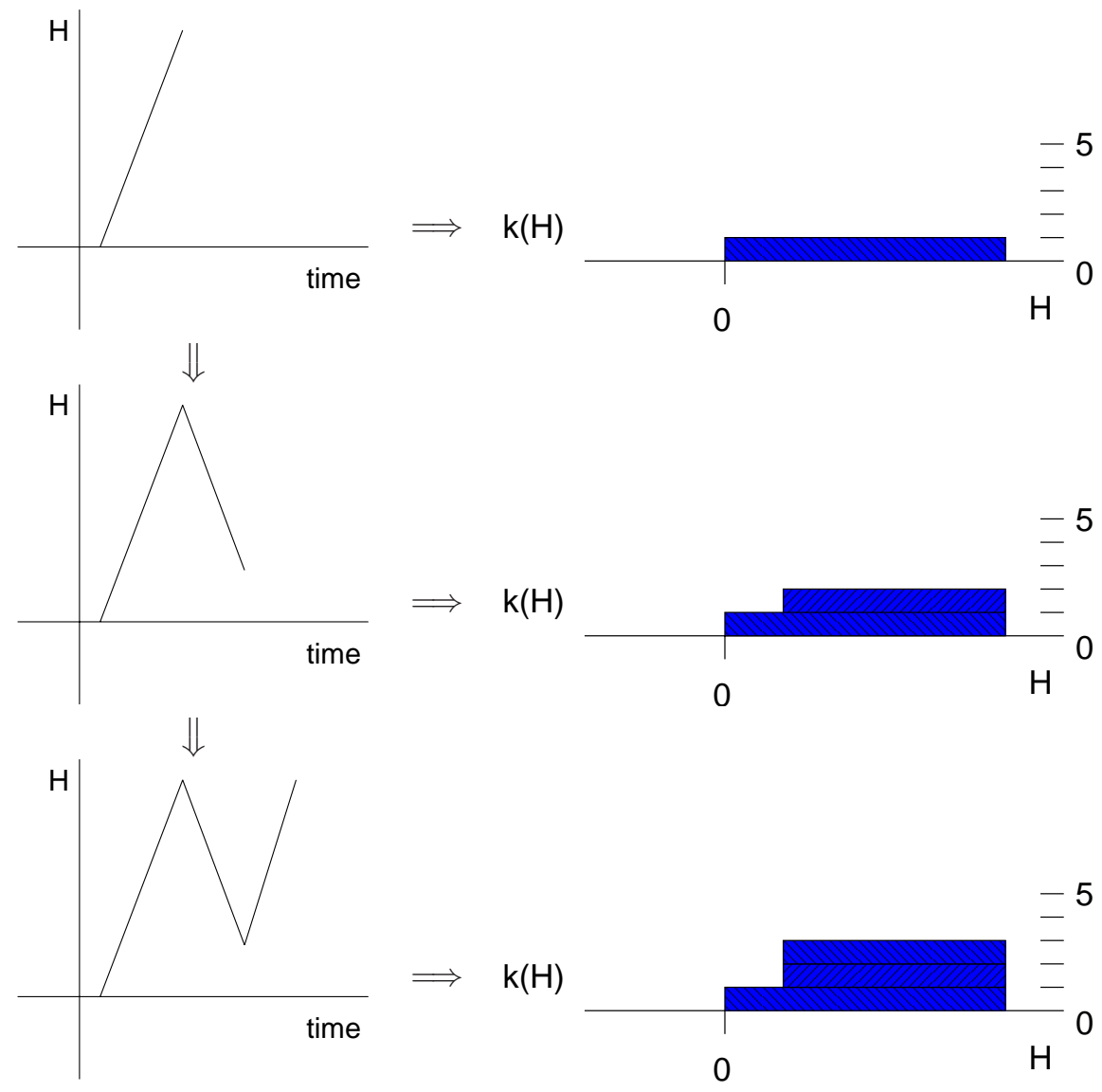

Figure 3: Depiction of the evolution of the counter $k$ as a function of the field $H$.

irreversible behavior - the switch of a moment's orientation from negative to positive or positive to negative. We therefore group these irreversible behaviors and reset (11) whenever the moment switches. This implies $N_{c} \times N_{I}$ separate instances of (11) must be employed, one for each combination of $H_{c}$ and $H_{I}$ quadrature points. In Section 3.2, we introduce an approximation to lessen this requirement.

\subsection{Implementation Issues}

The density in (10) is typically not known a priori. It may then appear that quadrature points and weights must be calculated for $\nu_{I}$ at each timestep. However, this is not the case. If $H_{I_{\text {nom }}}$ and $w_{I_{\text {nom }}}$ represent the quadrature points and products of quadrature weights and density heights $\left(w_{I}=\widehat{w}_{I} \cdot \nu_{I}\right)$ for the nominal density $\nu_{n o m}$, then the quadrature points and weights for $\nu_{I}$ can be calculated as

$$
H_{I}=H_{I_{\text {nom }}}(1+\alpha)^{\lambda^{k}}, \quad w_{I}=w_{I_{\text {nom }}} .
$$

Thus, the weights $w_{I}$ remain unchanged from the nominal density - the division in (10) cancels with the change in stepsize between quadrature points. The quadrature points are multiples of those used for the nominal density.

It is impossible to store the continuum required for (11). However, $H$ is bounded by material properties, allowing (11) to be discretized into an array of integer values. For a given input $H$, the closest value in the table is used. The size of the table therefore depends on the desired accuracy, but a few hundred values to a thousand values should be sufficient for most applications. Computational savings are achieved by realizing that $k$ is typically small. If we enforce $k \leq 255$, for example, one byte of storage can be used for each value. This suggests table sizes are less than one kilobyte, which is easily attainable on most platforms. Furthermore, the 
multipliers $(1+\alpha)^{\lambda^{k}}$ may be computed a priori and stored in a vector, leaving only an increment, a multiply, possibly a memory reset to be performed for each table at each timestep.

However, the theory still requires one table of $k$ values for each element in the coercive $\times$ interactive mesh. This can be reduced by approximating this with one table per interactive density quadrature point. This table is then shared across all the coercive field quadrature points and reset whenever the largest quadrature point is passed (Recall that the coercive distribution is bounded by a decaying exponential, so the largest quadrature point is finite). This will typically require only 15-80 tables of $k$ values, instead of potentially thousand.

\section{Model Attributes and Validation}

To illustrate attributes of the model, we summarize both simulation results and a model comparison with the nickel data plotted in Figure 1. To construct the model, parameters were initiall identified through a least squares fit to the data.

To illustrate the qualitative behavior of the model, the field shown in Figure 4(a) was input to the model to generate the magnetization plotted in Figure 4(b)-(d). The time-dependent plot in Figure 4(c) and expanded

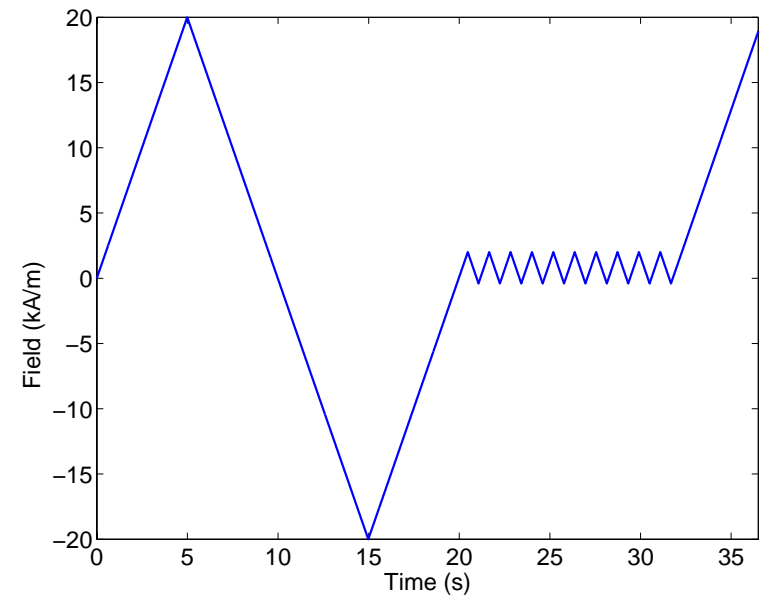

(a)

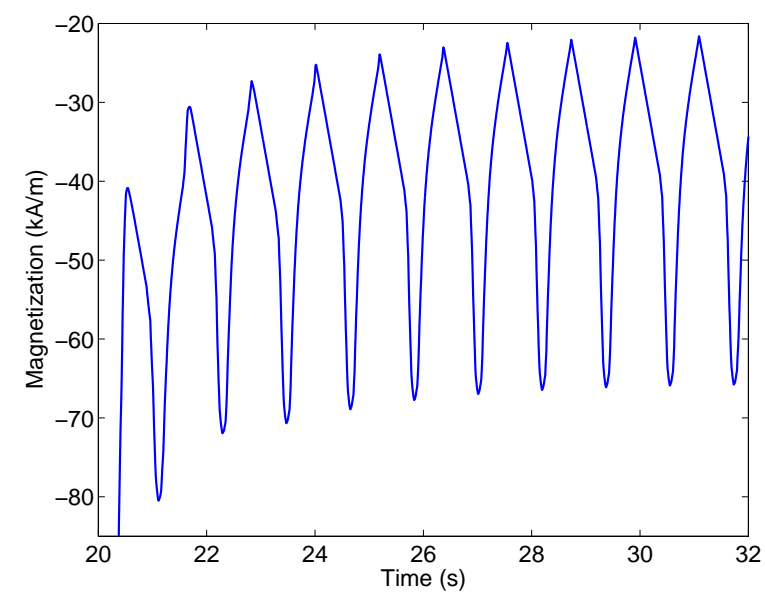

(c)

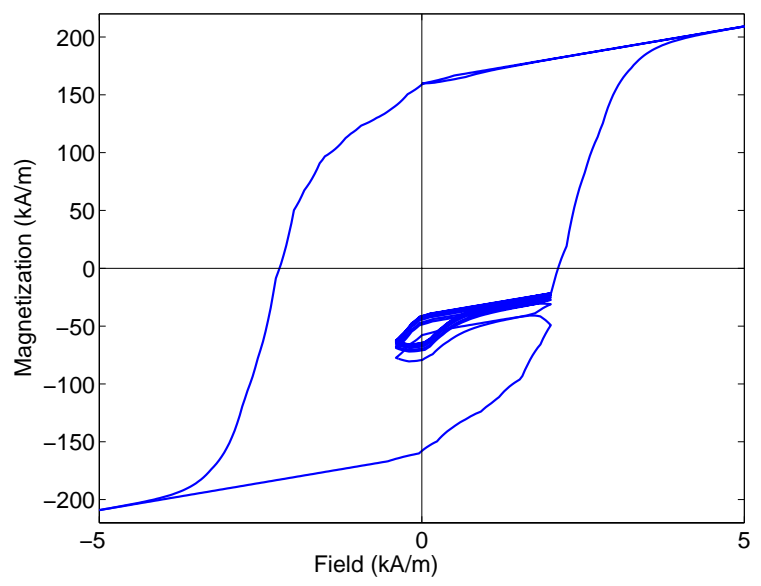

(b)

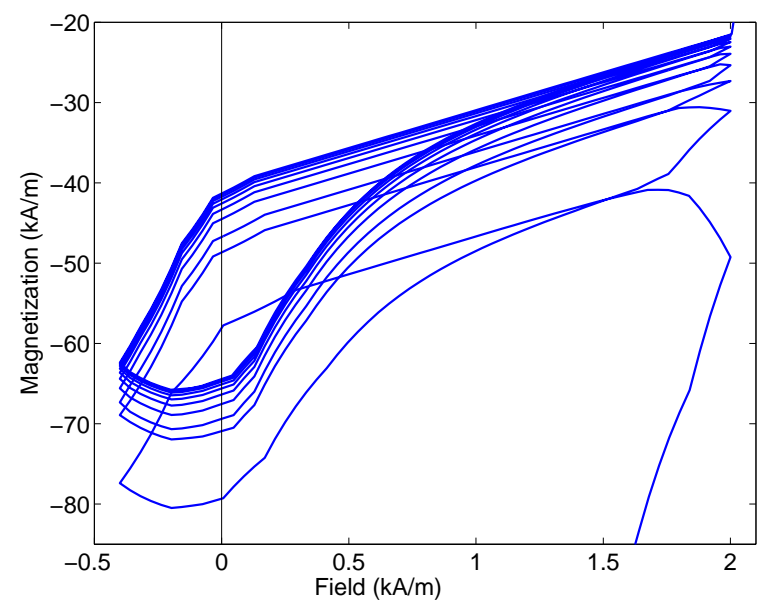

(d)

Figure 4: (a) Generated input, (b) and (d) magnetic field versus modeled magnetization, and (c) changes in the modeled magnetization as a function of time. 
perspective in Figure 4(d) illustrate the repration behavior that occurs during multiple minor loops traversals as well as the approach to a steady state minor loop. This qualitatively matches measured magnetic behavior (e.g., see [1].

The measured field shown in Figure 5(a) was subsequently input to the model to yield the major and minor loops shown in Figure 5(d) and (e). Comparison with the experimental values in Figure 5(b) and (c) - or, equivalently, Figure 1 - illustrate that the model is quantifying the measured magnetization behavior of the nickel sample.

\section{Concluding Remarks}

In this paper, we have extended the modeling framework of $[5,6,7]$ for characterizing ferromagnetic hysteresis to incorporate repration or accommodation effects. This is necessary both for fundamental material characterization and for quantifying the behavior of magnetic transducers employed as actuators or sensors in high drive regimes. These phenomena were incorporated by modifying the interaction field density to include the migration, approach to steady state behavior, and resetting as minor loops are traversed and exited. Aspects of the extended framework were illustrated through comparison with experimental nickel data.

\section{Acknowledgements}

The work of T.R.B. was supported by the United States Department of Education through a GAANN fellowship. The research of R.C.S. was supported in part by the Air Force Office of Scientific Research through the grants AFOSR-F49620-01-1-0107 and AFOSR-FA9550-04-1-0203 and the research of M.J.D. supported in part by Ohio State University.

\section{References}

[1] L.H. Bennett, F. Vajda, U. Atzmony, and L.J. Swartzendruber, "Accommodation study of a nanograin iron powder," IEEE Transactions on Magnetics, 32(5), pp. 4493-4495, 1996.

[2] T.R. Braun, R.C. Smith, and M.J. Dapino, "Experimental validation of a homogenized energy model for magnetic after-effects." CRSC Technical Report CRSC-TR05-26. Applied Physics Letters, submitted.

[3] S. Chikazumi, Physics of Ferromagnetism, 2nd Ed., Carendon Press, Oxford, 1997.

[4] B.D. Cullity, Introduction to Magnetic Materials, Addison-Wesley, Reading, MA, 1972.

[5] R.C. Smith, Smart Material Systems: Model Development, SIAM, Philadelphia, PA, 2005.

[6] R.C. Smith, M.J. Dapino, T.R. Braun and A.P. Mortensen, "A homogenized energy framework for ferromagnetic hysteresis," CRSC Technical Report CRSC-TR05-08. IEEE Transactions on Magnetics, to appear.

[7] R.C. Smith, M.J. Dapino and S. Seelecke, "A free energy model for hysteresis in magnetostrictive transducers," Journal of Applied Physics, 93(1), pp. 458-466, 2003.

[8] S.E. Zirka, Y.I. Moroz and E. Della Torre, "Combination hysteresis model for accommodation magnetization," IEEE Transactions on Magnetics, 41(9), pp. 2426-2431, 2005. 


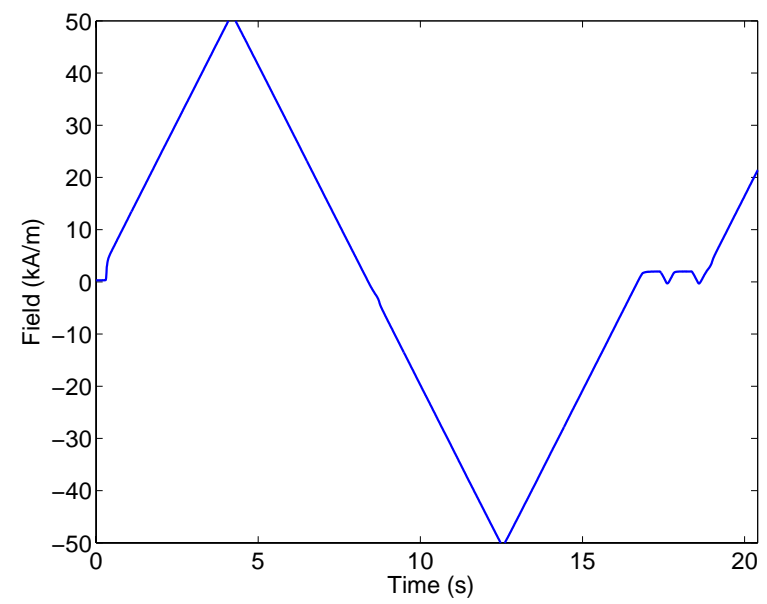

(a)

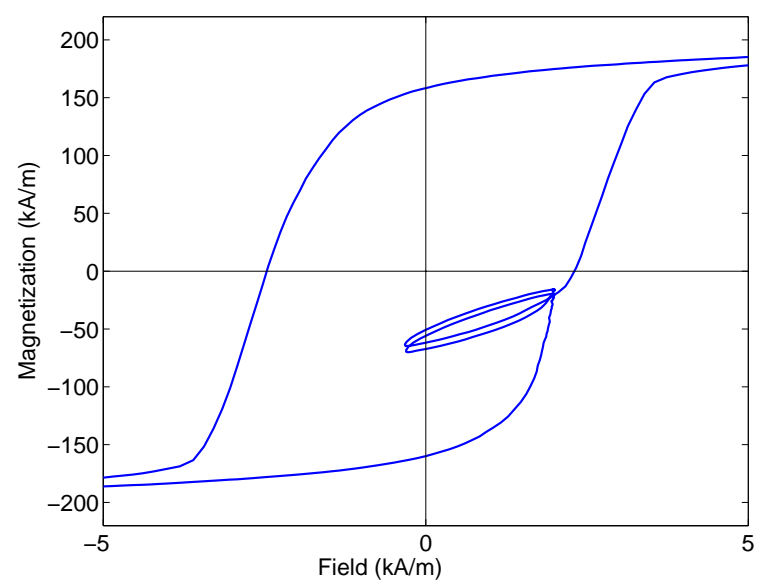

(b)

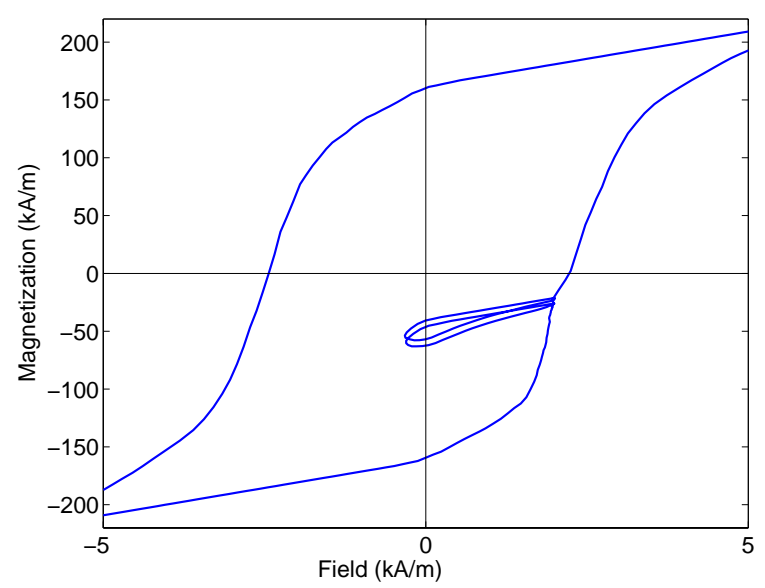

(d)

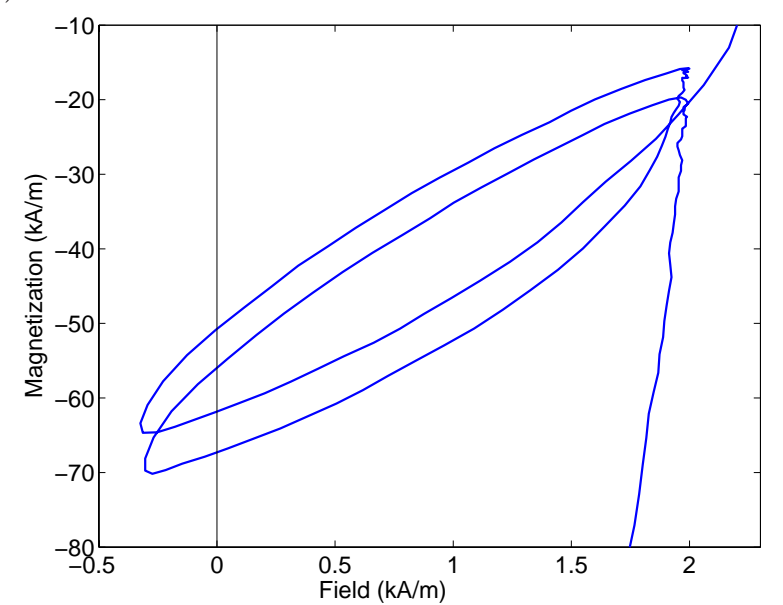

(c)

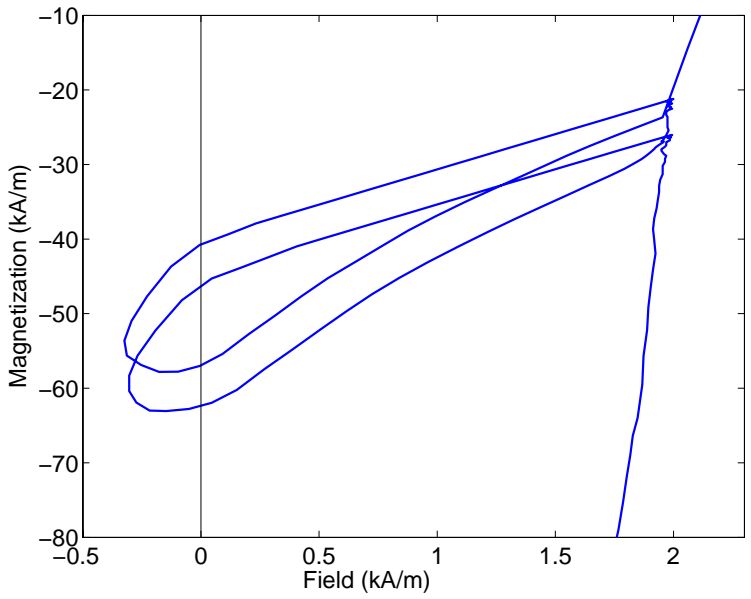

(e)

Figure 5: (a) Input magnetic field, (b) and (c) data measured from a nickel 200 rod, and (d) and (e) corresponding model fit. 